\title{
Contraste scalaire et aspectuel entre deux adverbes " complètement » et « tout à fait »
}

\author{
Chih-Ying Chiang* \\ Université Catholique Fujen, 24205 New Taïpei City, Taïwan
}

\begin{abstract}
Résumé. Rares sont les études linguistiques qui cherchent à identifier l'aspect impliqué dans des expressions adverbiales n'étant pas rangées traditionnellement dans la catégorie des adverbes de temps dont les adverbes de degré. Dans le présent travail, nous allons tenter de montrer, au travers des propriétés linguistiques, que complètement et tout à fait dits adverbes de degré, souvent traités comme des synonymes s'opposent du point de vue de l'expression du degré et de l'aspect. L'objectif du travail est, d'une part, d'établir les règles d'emploi de ces deux adverbes "synonymiques", et d'autre part, de clarifier leur divergence en termes de degré et d'aspect. Mots-clés : adverbe de degré, aspect, sémantique.
\end{abstract}

\begin{abstract}
Scalar and Aspectual Contrast between Two Adverbs "complètement" and "tout à fait". Few past linguistic studies sought to identify aspect contained in adverbs that were not classified as time adverbs. In this paper, we, via linguistic properties, attempt to show that complètement and tout à fait - called adverbs of degree and generally considered to be synonymous terms - differ in scale and aspect. The objective of the work is to establish the rules for the use of these two terms on one hand, and to clarify their differences in scale and aspect on the other. Keywords : adverbs of degree, aspect, semantics.
\end{abstract}

\section{Introduction}

Beaucoup de dictionnaires traitent complètement et tout à fait comme des synonymes, et utilisent l'un pour expliquer l'autre'. Cependant, on sait que la synonymie absolue n'existe pas. Il n'est donc pas étonnant de voir que la substitution de l'un des adverbes à l'autre n'est pas toujours heureuse :

(1) C'est (complètement + ? tout à fait) con!

Je suis (complètement + ? tout à fait) épuisé.

Zut ! J'ai (complètement + ? tout à fait) oublié notre rendez-vous.

(2) C'est (?? complètement + tout à fait) vrai, ce que vous dites.

Je me sens (*complètement + tout à fait) bien dans ce pays.

C'est (?? complètement + tout à fait) ce que je voulais dire.

*061991@mail.fju.edu.tw 
Quelles sont alors leurs règles d'emploi ? Lorsque la substitution est possible (ex. : Il est (complètement + tout à fait) guéri.), existe-t-il une différence sémantique entre ces deux expressions ? Ce genre de questions intéresse particulièrement les étudiants et les enseignants étrangers qui ne disposent pas d'une telle opposition adverbiale dans leur langue maternelle ${ }^{2}$.

Dans son mémoire de maîtrise, F.-F. Chen (1987) a étudié la différence de fonctionnement entre ces deux expressions adverbiales, en prenant principalement comme exemples des phrases où complètement modifie un verbe d'action (ex. : Il a complètement rempli cette bouteille.) alors que tout à fait modifie un adjectif (ex. : Il est tout à fait raisonnable, cet enfant!). D. Leeman \& C. Vaguer (1999) et V. Lenepeu $(2009,2013)$ ont également tenté de cerner l'identité de l'adverbe complètement; la recherche porte essentiellement sur les adjectifs dans le travail de D. Leeman \& C. Vaguer (ex. : Il est complètement nu.), et sur les verbes d'action dans les articles de V. Lenepeu (ex. : Ils contrôlent complètement certains villages. ). Les problèmes étudiés à des degrés divers dans ces travaux antérieurs sont la scalarité (ou le degré), la quantification/qualification, l'objectivité/subjectivité, l'aspect, entre autres. L'objectif du présent travail est de dégager les propriétés linguistiques de ces deux adverbes $\left(\mathrm{cf} .\left(\mathrm{P}_{1}\right)-\left(\mathrm{P}_{7}\right)\right)$ plus particulièrement en termes de scalarité et d'aspect, en prenant en compte des structures phrastiques et des types d'adjectifs les plus variés possibles, afin d'avancer vers une description du fonctionnement de ces deux adverbes qui se base sur d'autres preuves linguistiques.

\section{Complètement et tout à fait sont-ils des adverbes de degré ?}

\section{1 État des lieux}

On distingue traditionnellement trois degrés de force. Dans Le bon usage, il s'agit des degrés faible (ex. : à peine, peu, un peu, etc.), moyen (ex. : pas mal, assez, etc.) et haut (ex. : très, beaucoup, grandement, infiniment, etc.) ; complètement et tout à fait y sont rangés

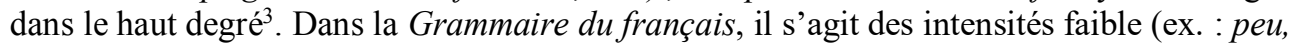
faiblement, légèrement, etc.), moyenne (ex. : assez, moyennement, etc.) et élevée (ex. : très, fort, etc.) ; complètement et tout à fait y sont également regroupés ensemble dans l'intensité élevée ${ }^{4}$.

Comme la plupart des grammairiens, D. Lee méthodique man \& C. Vaguer considèrent ${ }^{5}$ complètement comme un indicateur de degré haut, mais affirment de plus que ce degré est " extrême", en raison de l'opposition entre nu en caleçon et complètement nu. Partant de l'idée que l'adjectif indique "une propriété ou un état mais sans exprimer par lui-même que la qualité en question atteint son degré maximal », les deux auteurs pensent que complètement va «dans le sens potentiellement impliqué (par défaut, pourrait-on dire) mais cette implication n'est justement pas actualisée sans l'adverbe ${ }^{6}$. La possibilité d'énoncer nu en caleçon (cf. (3)) montre que l'adjectif lui-même ne désigne pas forcément le plus haut degré de la qualité en question.

(3) J'ai des souvenirs charmants d'après-midi [...] sous la petite avenue de peupliers, nu en caleçon, avec l'odeur des filets et du goudron. (Flaubert)

À l'inverse, dans une phrase comme Il est complètement nu, c'est complètement qui actualise "pleinement" la propriété être $n u$. 
Concernant le degré, un autre point dans le travail de D. Leeman \& C. Vaguer mérite d'être mentionné. Elles estiment que complètement lui-même est gradable, au vu des exemples attestés suivants qui acceptent des modificateurs de degré tels que le plus et très:

(4) D'un rêve à un autre, il n'est pas rare que le fait d'appartenir également à un enchaînement d'images né du sommeil soit le caractère le plus complètement insignifiant. (Graq)

(5) La marine américaine avait en service ou en construction [...] trente navireateliers, dont treize spécialisés dans les grosses réparations de coque, très complètement équipés. (Le Masson)

\subsection{Notre point de vue et les arguments}

Intuitivement parlant, nous dirions que complètement peut fonctionner comme un adverbe de degré alors que ce n'est pas le cas de tout à fait. Cette première intuition se justifie par trois types de phénomène, $\left(\mathrm{P}_{1}\right)-\left(\mathrm{P}_{3}\right)$ ci-dessous, qui montrent le comportement opposé de ces deux expressions adverbiales quand il s'agit d'indiquer un degré.

$\left(\mathrm{P}_{1}\right)$ Complètement peut s'enchaîner avec un autre adverbe de degré, un peu ou assez, dans la suite $p$, et même $q$, alors que tout à fait a du mal à y entrer. Selon O. Ducrot (1980), même relie deux arguments ( $p$ et $q$ ) placés sur une même échelle, dont le second est toujours argumentativement plus fort que le premier par rapport à une conclusion identique $(r)$. C'est pourquoi on a (6a), et non (6b) :

(6a) Léo est un peu égoïste, et (parfois) même très égoïste : ne le prends pas dans notre groupe (r).

(6b) * Léo est très égoïste, et (parfois) même un peu égoïste : ne le prends pas dans notre groupe ( $r$ ).

Un peu, assez et très représentent la force argumentative en ordre croissant :

(7) Il est un peu égoïste, et je dirais même assez égoïste.

(8) Il est assez égoïste, et même très égoïste.

Nous constatons que complètement entre sans difficulté dans cette structure en occupant la place $q$, comme très, lorsqu'il est couplé avec un peu et assez :

(9) Je suis un peu dégoûtée de mon travail, et même (très + complètement) dégoutée.

(10) La soupe est assez froide, et même (très + complètement) froide.

Les exemples (9) et (10) permettent de ranger complètement dans le même groupe que les adverbes traditionnellement traités comme indicateurs de degré, et, de ce fait, justifient l'opinion qu'il désigne un degré supérieur. Quant à tout à fait, bien qu'il puisse dans certains cas, cf. (11)-(14), avoir la même distribution que un peu, assez et très pour modifier un adjectif,

(11) Ce sport est (un peu + assez + très + tout à fait) fatigant.

(12) Cette infirmière est (assez + très + tout à fait) gentille avec moi.

(13) Je suis (un peu + assez + très + tout à fait) habitué à ce rythme de travail.

(14) C'est un homme (assez + très + tout à fait) sincère.

son enchaînement avec un peu ou assez dans la formule $p$, et même $q$ pour modifier les mêmes adjectifs paraît maladroit. Or, très s'y insère sans aucun problème :

(11a) Ce sport est (un peu + assez) fatigant, et même (très + ? tout à fait) fatigant.

(12a) Cette infirmière est assez gentille avec moi, et même (très + ? tout à fait) gentille. 
(13a) Je suis (un peu + assez) habitué à ce rythme de travail, et même (très + ? tout à fait) habitué.

(14a) C'est un homme assez sincère, et même (très + ? tout à fait) sincère.

Les comportements différents de complètement et de tout à fait dans les exemples ci-dessus confortent notre intuition selon laquelle tout à fait ne désigne pas un degré, contrairement à complètement.

$\left(\mathrm{P}_{2}\right)$ Face à certains termes non-gradables ${ }^{7}$, complètement réagit comme assez et très alors que tout à fait se comporte différemment :

(15) $\quad C$ 'est (*assez + *très $+*$ complètement + tout à fait) ça.

(16) C'est (*assez + *très $+{ }^{*}$ complètement + tout à fait) ce que je voulais dire.

Le troisième type de phrases qui justifie la distinction entre complètement et tout à fait quand il s'agit d'indiquer un degré contient une série d'adjectifs qualitatifs que J.-C. Anscombre (1991) appelle adjectifs qualifiants extrinsèques, qu'il oppose aux qualifiants intrinsèques. La notion d'adjectif qualifiant vient à l'origine de J.-C. Milner (1978), selon qui les classifiants dénotent une classe stable, objective et permettent l'attribution d'une propriété indépendamment de l'opinion du locuteur, alors que les qualifiants sont liés à un acte d'énonciation particulier de la part du locuteur. Ainsi, rouge s'oppose à délicieux lorsqu'il s'agit du vin. Quant à la distinction entre qualifiants intrinsèques et qualifiants extrinsèques, elle se situe au niveau du degré de la propriété : «les qualifiants intrinsèques renvoient à un simple degré éventuellement élevé sur l'échelle associée au substantif» tandis que "les qualifiants extrinsèques désignent un (très) haut degré »" ${ }^{8}$. Les qualifiants intrinsèques sont par exemple bon, fort, grand, mauvais, vif, etc. qui s'opposent aux qualifiants extrinsèques tels que délicieux, excellent, extraordinaire, merveilleux, magnifique, remarquable, catastrophique, désastreux, lamentable, minable, etc. Ces derniers sont sémantiquement très proches de l'interjection ${ }^{9}$. Suivant cette distinction, nous remarquons que :

( $\left.\mathrm{P}_{3}\right)$ Tout à fait s'associe aisément avec les adjectifs qualifiants extrinsèques, qui, selon nous, marquent par eux-mêmes le degré extrême, tandis que complètement est exclu de ce type d'association. L'une des propriétés des qualifiants extrinsèques, observées par J.-C. Anscombre (2013), est qu'ils n'admettent pas la combinaison avec très, cf. (17)-(25). La raison en est que les adjectifs comportant intrinsèquement le plus haut degré ne sont pas en mesure d'être gradués car le degré extrême est partie constituante de la nature de ce type d'adjectifs, qui n'est pas compatible avec la gradation. Par conséquent, les qualifiants extrinsèques ne sont pas gradables. Il est intéressant de voir que tout à fait s'emploie sans problème avec eux, alors que ce n'est absolument pas le cas de complètement :

(17) Il m'a préparé un plat $(*$ très $+*$ complètement + tout à fait) délicieux.

(18) Il a proposé un projet (*très $+*$ complètement + tout à fait) excellent.

(19) Il a fait une présentation (*très $+*$ complètement + tout à fait) extra.

(20) Le candidat a fait un exposé (*très $+{ }^{*}$ complètement + tout à fait) extraordinaire.

(21) Ce tableau ancien reste dans un état (*très $+*$ complètement + tout à fait) merveilleux.

(22) Le chanteur a une voix (*très $+*$ complètement + tout à fait) magnifique.

(23) Le président a fait un discours $(*$ très $+*$ complètement + tout à fait) remarquable.

(24) Le bilan est $(*$ très $+*$ complètement + tout à fait) catastrophique.

(25) C'est $\left(^{*}\right.$ très $+{ }^{*}$ complètement + tout à fait) minable, ce que tu fais ${ }^{10}$. 
Les phrases en (17)-(25) ramènent une fois de plus complètement du côté des adverbes de degré, et le séparent de tout à fait. Il convient donc de considérer que tout à fait ne désigne pas un degré, contrairement à ce qu'on croit habituellement.

\section{Nos hypothèses}

Après les premières observations présentées en $\S 2$, nous émettons l'hypothèse que le fonctionnement de complètement relève de l'ordre quantitatif tandis que celui de tout à fait relève de l'ordre qualitatif.

F.-F. Chen et V. Lenepveu formulent aussi une hypothèse quantitative pour complètement. Mais tandis que l'une souligne l'aspect du procès sur lequel porte l'adverbe ${ }^{11}$, l'autre met l'accent sur la complétude du référent ${ }^{12}$. Nous défendons l'hypothèse que ces deux valeurs sont réductibles à une seule fonction sémantique au travers de la notion de quantification. Voici ce que nous entendons par la quantification opérée par complètement : la quantification marquée par complètement se caractérise par des phénomènes processifs, qui se manifestent soit par la marque ou l'implication de l'accomplissement d'un procès (par exemple, J'ai complètement oublié notre rendez-vous et La soupe est complètement froide représentent respectivement ces deux cas de figure), soit par la fin du parcours de tous les éléments/facteurs présents dans le sujet (par exemple, après avoir parcouru les substances constituant le produit et vérifié une à une leur recyclabilité, on peut dire Ce produit est complètement recyclable). Il se peut, par ailleurs, que ces deux phénomènes se réalisent dans une même occurrence. C'est le cas de Mes rosiers sont complètement morts, qui peut signifier à la fois qu' " il n'y a plus moyen de sauver mes rosiers » et que « tous les rosiers sont morts ».

Quant à tout à fait, nous dirions qu'il exprime une confirmation. Il confirme la correspondance entre ce qui se passe dans une situation et le procès où apparaît tout à fait. Ce procès est souvent un verbe d'état (être, avoir l'air, devenir, pouvoir, correspondre à, etc. $)^{13}$. Ces hypothèses sur complètement et tout à fait seront vérifiées sous l'angle de l'aspect.

\section{Complètement et tout à fait impliquent-ils un aspect ?}

\section{1 État des lieux}

Tout à fait n'est pas examiné par F.-F. Chen du point de vue de l'aspect. Quant à complètement, F.-F. Chen et V. Lenepveu ont remarqué qu'il peut porter sur un verbe télique. C'est dans l'article de D. Leeman \& C. Vaguer que le rapport entre complètement et l'aspect est explicité : complètement "marque moins la quantité que l'aspect» et 《implique un aspect duratif $»^{14}$. Cette hypothèse est faite à la suite de l'observation des séquences telles que être malade, être fou, être chauve, etc. qui représentent une propriété. Une propriété, s'opposant à un événement (ex. : avoir chanté, être parti, etc.), est généralement vue comme étant stative et durative. Un très grand nombre de prédicats du type être $+A d j$., appelés dans ce travail prédicats adjectifs, ne peuvent guère être modifiés par complètement, comme ce qu'illustre (26), d'autant que la liste des adjectifs en (26) n'est pas finie :

$$
\begin{aligned}
& \text { Notre nouveau stagiaire est complètement }(* \text { beau }+* \text { étrange }+* \text { intelligent } \\
& \left.+* \text { jeune }+* \text { joyeux }+* \text { paresseux }+* \text { poli }+{ }^{*} \text { sportif }+* \text { sympathique }\right) .
\end{aligned}
$$


Il est donc nécessaire de regrouper les propriétés selon des critères bien définis. Une classification au travers des prédicats du type être + Adjectif a été faite dans J.C. Anscombre (2010). Nous nous appuierons sur ce classement pour faire ressortir la différence des aspects impliqués dans complètement et tout à fait à travers les types de prédicat adjectif compatibles avec ces deux expressions adverbiales.

\subsection{Propriétés intrinsèque/extrinsèque, essentielle/accidentelle}

J.-C. Anscombre distingue deux grands types de propriétés : intrinsèque et extrinsèque. Une propriété intrinsèque représente un trait définitoire, constitutif de l'entité en question, par exemple : la couleur naturelle des cheveux d'un individu (en énoncé, on a Il est blond ou C'est un blond), les traits qui définissent la voiture (on a des phrases génériques comme La voiture est rapide, La voiture a un volant, un moteur et quatre roues), etc. Une propriété est extrinsèque si elle correspond à l'état contingent où se trouve l'entité considérée. Elle ne définit pas l'entité en tant qu'individu, mais en tant que participant à un procès, par exemple : la couleur des cheveux teints d'un individu, l'état de la voiture après un accident (ex. : La voiture est abimée ou encore La voiture est en panne ${ }^{15}$ ), etc.

L'entité en question peut être un élément unique, un groupe d'éléments ou bien une classe entière d'éléments. Il s'ensuit qu'une propriété intrinsèque de l'entité peut être essentielle ou accidentelle: "une propriété est essentielle pour une classe C si elle caractérise toute la classe, et accidentelle si elle ne caractérise qu'une partie de la classe. ${ }^{16}$ Ainsi, l'énoncé La voiture est rapide relève-t-il d'une propriété intrinsèque, mais aussi essentielle du sujet. En revanche, La voiture est électrique renvoie à une propriété intrinsèque et accidentelle du sujet. De même, une propriété extrinsèque peut être subdivisée en extrinsèque essentielle et extrinsèque accidentelle. $\hat{E}$ tre responsable de la pollution est une propriété essentielle de la classe des voitures tandis que être en panne est une propriété accidentelle pour la même classe.

En d'autres termes, les propriétés intrinsèques désignent en quelque sorte l'état d'origine de l'entité puisqu'il est définitoire de l'entité en question, alors que les propriétés extrinsèques représentent un état acquis de l'entité, issu d'un procès. De ce fait, les propriétés intrinsèques dénotent un état qui est, d'après nous, purement statif, tandis que les propriétés extrinsèques renvoient à l'état qui résulte d'un procès. Nous verrons dans $\S 4.3$. que tout à fait dans les phrases comportant un prédicat adjectif tend à exprimer un simple état, contrairement à complètement qui dénote régulièrement un état acquis.

\subsection{Complètement, tout à fait et les types de propriété}

(P) Un groupe d'exemples comportant des adjectifs non-participiaux montre que complètement se combine aisément avec la propriété extrinsèque d'une entité, et refuse systématiquement la propriété intrinsèque de la même entité :

(27) Le café est complètement (* chaud + froid $)^{17}$.

(28) La soupe est complètement (*chaude + froide).

(29) Le patron est complètement (*coléreux + furieux).

(30) Son patron est complètement (*alcoolique + ivre + soûl). Son patron est complètement $(* \text { maladif }+ \text { malade })^{18}$.

Le café se définit comme une boisson chaude, et se boit chaud, comme d'ailleurs la soupe. Etre chaud(e) est donc l'état initial du café et de la soupe. Il est assez étrange de décrire le café et la soupe par complètement chaud(e). En revanche, complètement froid(e) peut parfaitement qualifier leur état après qu'ils ont connu un refroidissement ${ }^{19}$. Signalons au 
passage que si être froid relève d'une propriété intrinsèque d'une entité, la qualification par complètement froid ne convient plus :

(28a) L'hiver, il fait toujours (très $+*$ complètement) froid dans ce pays.

Coléreux est le tempérament d'un individu, donc la nature psychique de la personne, alors que furieux correspond à l'état de la personne face à un événement déplaisant, d'où la parfaite combinaison de complètement furieux. On peut, d'ailleurs, facilement insérer en (29) une séquence précisant l'événement en question; cette insertion ne changera nullement la différence d'acceptabilité entre *complètement coléreux et complètement furieux:

(29a) Vu l'arrogance de sa petite-fille, la grand-mère était complètement (*coléreuse + furieuse).

Cette opposition intrinsèque/extrinsèque se retrouve également entre les adjectifs en (30) et en (31). Etre alcoolique représente un trouble du comportement, c'est-à-dire un défaut permanent de la personne. Être maladif décrit une facilité constante à tomber malade ou signale l'existence d'une maladie chronique. Ces deux prédicats constituent des traits permettant de caractériser un individu, ils font donc partie des propriétés intrinsèques de la personne. En revanche, être ivre, être soûl et être malade décrivent un état contingent et provisoire de la personne après l'accomplissement d'un procès, i.e. boire et perdre la raison par exemple. À cette série d'exemples comportant un prédicat adjectif, il est légitime d'ajouter (32) :

(32) Ce matin, Léo avait les yeux complètement (*bleus + rouges).

Bien qu'avec la nouvelle technologie on puisse avoir de nos jours des lentilles bleues qui donnent une couleur artificielle des yeux, bleu est traditionnellement vu comme une couleur naturelle des yeux de l'être humain. Ce n'est absolument pas le cas de rouge, qui représente l'état des yeux après une infection, par exemple.

(P5) Sa grande affinité avec les adjectifs verbaux formés sur le participe passé témoigne de la valeur processive de complètement ${ }^{20}$. Ce type d'adjectifs dénote lexicalement l'accomplissement d'un procès. Leur grande affinité avec complètement milite en faveur de l'hypothèse que complètement implique l'idée de procès :

(33) C'est un miracle! La tumeur est (complètement + ? tout à fait) disparue ${ }^{21}$.

(34) Après l'incendie, le chalet est (complètement $+*$ tout à fait) réduit en cendres. Il n'en reste plus rien.

(35) La maison est (complètement + ? tout à fait) nettoyée.

(36) Après plusieurs jours de pluie, le village est (complètement $+*$ tout à fait) inondé.

(37) J'ai fait tomber la tasse. Elle est (complètement + *tout à fait) cassée.

(38) La Mairie a décidé de rénover le quartier. Le grand immeuble place St. Amour sera (complètement $+*$ tout à fait) détruit.

Tout à fait dans ces phrases ne sera pas le premier choix, et paraît plus ou moins instable. Mais son association avec ce type d'adjectifs ne semble pas pour autant totalement exclue. Dans le cas où le locuteur confirme l'état de la situation, tout à fait aura la possibilité d'être employé :

A : Vous êtes sûr qu'on peut arrêter le traitement?

B : Sûr et certain. Vous êtes guéri. La tumeur est tout à fait disparue.

Tout à fait ne peut venir qu'en confirmation du procès qui doit être présent dans la question et la réponse. 

A : La maison est-elle bien nettoyée?
B : Oui, elle est, je te l'assure, tout à fait nettoyée!

C'est d'ailleurs la raison pour laquelle tout à fait peut s'employer seul pour donner une confirmation :
A : La tumeur est disparue?
$\mathrm{B}:$ Tout à fait!
A : La maison est-elle bien nettoyée?
B : Tout à fait !

Néanmoins, nous rencontrons des exemples gênants où tout à fait porte sans problème sur un adjectif formé sur le participe passé :

(39) Je suis tout à fait habitué à ce rythme de travail.

(40) Je suis tout à fait débarrassé de mes soucis.

(41) L'enfant est tout à fait consolé de son chagrin.

(42) L'enfant est tout à fait (rétabli + guéri + remis sur pied).

(43) L'enfant est tout à fait rassuré.

(44) Le spectacle est tout à fait réussi.

(45) Quand la peinture sera tout à fait (finie + achevée), on la mettra à sécher.

Les linguistes ${ }^{22}$ s'intéressent aux problèmes des adjectifs formés sur les participes reconnaissent qu'il y a encore beaucoup à faire pour établir la régularité sémantique de ce type d'adjectifs. En effet, "les exceptions » rencontrées sont « infiniment plus nombreuses que les cas réguliers ", écrit J.-C. Anscombre (2000: 241), qui estime que l'interprétation des adjectifs verbaux participes dépend certes du verbe dont ils sont issus, mais aussi du nom auquel ils s'associent. Suivant ce principe, les adjectifs en (39)-(42) sont issus des verbes pronominaux (s'habituer à, se débarrasser de, se consoler de, s'établir, se mettre sur pied); réussi, fini et achevé sont dérivés d'un verbe transitif (réussir le spectacle, finir/achever la peinture). Guéri et rassuré sont plus compliqués car ils peuvent, chacun, avoir deux constructions (L'enfant a guéri / On a guéri l'enfant ; L'enfant s'est rassuré / On a rassuré l'enfant). Or, le lien entre l'adjectif participe et son verbe dérivé ne semble pas nous permettre d'avoir une explication de l'usage de tout à fait dans ces exemples.

Il est possible que, malgré le fait que ces participes passés marquent l'accomplissement d'un processus, on entende dans ces énoncés un sens qui met l'accent sur l'état : Je suis tout à fait habitué à ce rythme de travail a le sens de « Maintenant j'ai l'habitude », Je suis tout à fait débarrassé de mes soucis a le sens de " Maintenant je suis serein », L'enfant est tout à fait (rétabli + guéri + remis sur pied) a le sens de « Maintenant l'enfant est en bonne santé », Quand la peinture sera tout à fait (finie + achevée) a le sens de «Quand la peinture sera un vrai tableau, plus un chantier », etc.

(P6) Plusieurs travaux antérieurs soutiennent que les adjectifs verbaux formés sur le participe présent, possédant la valeur aspectuelle du présent, désignent «l'action concomitante, c'est-à-dire qui se développe en même temps que celle du verbe de la proposition où il se trouve $»^{23}$, et renvoient à une qualité inhérente de l'entité. Nous constatons qu'à la différence de complètement, tout à fait semble avoir une plus grande affinité avec ce type d'adjectifs verbaux :

(46) C'est un homme (*complètement + tout à fait) charmant.

(47) C'est une histoire (*complètement + tout à fait) amusante.

(48) C'est un sport (*complètement + tout à fait) fatigant.

(49) Il nous a fait une proposition (*complètement + tout à fait) séduisante.

(50) Il a présenté un projet (*complètement + tout à fait) intéressant. 
À ce stade, il faudrait signaler un emploi très familier de complètement (marqué par le signe \#) qui est jugé sous standard par mes informateurs natifs. Cf. les exemples suivants ${ }^{24}$ :

(51) L'histoire est (\# complètement + tout à fait) (traumatisante + effrayante + impressionnante + choquante + démoralisante + affolante + délirante). La question est (\# complètement + tout à fait) (embarrassante + gênante).

Cet emploi oral sous standard donne une certaine valeur exclamative à la phrase, donc s'entend en principe dans des phrases courtes comme C'est complètement traumatisant! Après notre analyse supra, on peut comprendre en quoi cet usage de complètement transgresse la loi. Dans une phrase plus longue à la syntaxe plus élaborée où l'adjectif -ant représente un élément définitoire de l'entité, cet usage familier de complètement n'a plus lieu d'être :

Pendant la guerre, il y a eu des massacres (*complètement + tout à fait) traumatisants.
Une épidémie meurtrière (*complètement + tout à fait) effrayante.
Ces petits fours sont (*complètement + tout à fait) appétissants.
Cette offre est (*complètement + tout à fait) alléchante.
On a eu un feu d'artifice (*complètement + tout à fait) impressionnant.

Notons que l'usage de complètement en (58) ne pose aucun problème. La raison en est qu'un travail peut être jugé de différents aspects.

(58) Le travail est (complètement + tout à fait) satisfaisant.

$\left(\mathbf{P}_{7}\right)$ Une dernière propriété qui sépare ces deux adverbes: à la différence de complètement, tout à fait a une grande affinité avec les adjectifs en -able/-ible qui marquent par eux-mêmes la valeur purement stative, sans l'intervention d'un procès.

(59) Ce tissu est (? complètement + tout à fait) nettoyable.

(60) Il a fait un discours (*complètement + tout à fait) remarquable.

(61) Attention! Il est (*complètement + tout à fait) capable de nous trahir.

(62) C'est (*complètement + tout à fait) compréhensible que les enfants s'ennuient dans la classe.

(63) Il est (*complètement + tout à fait) possible qu'on supprime cette subvention du jour au lendemain.

Le (64) ne sera pas un contre-exemple. L'usage de complètement est possible lorsqu'il s'agit d'une machine démontable dont on peut nettoyer toutes les pièces. Complètement indique la fin du nettoyage de toutes ces pièces.

(64) La machine est (complètement + tout à fait) nettoyable.

On trouve aussi des cas où complètement modifie un adjectif en -ible dans des énoncés courts, cf. (65)-(66). Ces formules sont sous standard :

(65) C'est (\# complètement + tout à fait) inadmissible.

(66) C'est (\# complètement + tout à fait) incompréhensible.

Nous remarquons qu'avec un adjectif en -able/-ible de sens négatif - i.e. inadmissible, incompréhensible, incapable, inacceptable, invérifiable, immangeable, impossible, etc. complètement s'accorde mieux qu'avec les adjectifs correspondant de sens affirmatif, bien que tout à fait reste le meilleur choix. D'ailleurs, lorsque complètement se combine avec un adjectif en -ant, il semble avoir aussi une préférence des adjectifs à valeur négative, cf. (51) et (52) mentionnés supra. 


\section{Conclusion}

En guise de conclusion, quelques points à mentionner. Premièrement, l'hypothèse concernant l'opposition aspectuelle entre complètement et tout à fait est à peu près confirmée, malgré quelques difficultés rencontrées avec certains adjectifs participiaux. De ce fait, Si l'on admet que complètement s'accorde avec des phénomènes processifs marquant l'accomplissement du procès, on peut facilement comprendre pourquoi on accepte sans aucune réserve la phrase La bouteille est complètement vide, et non la phrase ? La bouteille est complètement pleine. Ce genre de contraste montre par ailleurs comment le substantif en français est stéréotypiquement perçu en langue: dans le cas précis, le stéréotype de la bouteille est « être remplie d'un liquide».

Deuxièmement, certaines propriétés linguistiques montrent que tout à fait se révèle un adverbe de confirmation plutôt que de degré. Nous remarquons, d'ailleurs, que lorsqu'il se trouve dans un contexte favorable à l'expression de la confirmation, par exemple dans une réplique ou une réponse de confirmation, il semble se libérer de la contrainte lexicale concernant la combinaison avec les adjectifs formés sur les participes passés. Les associations être tout à fait épuisé et être tout à fait nettoyé sont a priori maladroites. Mais leur énonciation ne pose plus aucun problème dans les dialogues comme suit :

A : Tu as l'air un peu fatigué, mais peut-être pas au point d'être épuisé. Tu pourrais peut-être donner le bain à Léo?

B : Arès une grosse journée avec un patron difficile, je suis, je te l'assure, tout à fait épuisé 25 !

A : La maison est-elle bien nettoyée?

B : Oui, elle est, je te l'assure, tout à fait nettoyée!

On a vu aussi que tout à fait admet mal d'être introduit par même dans la suite $p$, et même $q$ (cf. (11a)-(14a)). Cependant, son enchaînement avec même devient possible s'il est employé dans une réponse exprimant une confirmation :
A : Tu ne trouves pas que ce sport est un peu fatigant?
B : Ah si, je dirais même qu'il est tout à fait fatigant.
A : L'infirmière a été gentille avec toi ?
$\mathrm{B}$ : Ah oui, je dirais même qu'elle a été tout à fait gentille avec moi.

Enfin, il semble que la différence sémantique entre complètement et tout à fait s'estompe, voire disparaisse dans des phrases négatives (ex. : La situation n'est pas (complètement + tout à fait) maîtrisable.), ce phénomène mérite d'être exploité, ce que nous n'avons pas pu faire dans ce travail.

Je remercie vivement Joëlle Cailleaux, Jean-Yves Heurtebise, Françoise Metzger, Éric de Payen et André Reichard, qui m'ont servi d'informateurs, et en particulier Catherine Nambride qui a lu le manuscrit de cet article et avec qui j'ai eu de nombreuses discussions fructueuses.

\section{Références bibliographiques}

ANSCOMBRE J.-C. (1991). La déterminant zéro : quelques propriétés. Langages, 102, 103-124.

ANSCOMBRE J.-C. (1995). Morphologie et représentation événementielle : le cas des noms de sentiment et d'attitude. Langue française, 105, 40-54.

ANSCOMBRE J.-C. (2000). Un problème de sémantique lexicale : l'interprétation active/passive des adjectifs verbaux participes en position d'épithète. Le passif «Actes du colloque international, Institut d'Etude Romanes, Université de Copenhague, du 5 au 7 mars 1998 », 237-259.

ANSCOMBRE J.-C. (2010). États et propriétés dans les combinaisons « être + Adjectif ». Le français moderne, 2, 186-205. 
ANSCOMBRE J.-C. (2013). Les exclamatives : intensification ou haut-degré. Langue française, 177, 23-36.

ARRIVÉ M., GADET F. \& GALMICHE M. (1986). La grammaire d'aujourd'hui. Paris : Flammarion.

CHEN F.-F. (1987). Étude comparative de " tout à fait », " complètement » et «tout ». Mémoire de maîtrise. Université de Franche-Comté.

DUCROT O. (1980) Les échelles argumentatives. Paris : éditions de Minuit.

ERNOUT A. \& THOMAS F. (1972). Syntaxe latine. Paris : Klincksieck.

GREVISSE M. (2001). Le bon usage, $13^{\mathrm{e}}$ édition. Duculot.

KUPFERMAN L. (1991). L'aspect nominal et l'extraction en « en ». Français moderne, 59, 2, 113 147.

KUPFERMAN L. (1992). Une morphologie dispositionnelle. De la musique à la linguistique, hommages à Nicolas Ruwet, L. Tasmowski \& A. Zribi-Herts, eds., Communication et Cognition, 345-359.

LEEMAN D. (1987). À ma grande surprise. Revue québécoise de linguistique, 1, 2, 415-434.

LEEMAN D. (1995). Pourquoi peut-on dire « Max est en colère » mais non « Max est en peur »? Hypothèses sur la construction «être en $\mathrm{N}$ ». Langue française, 105, 55-69.

LEEMAN D. \& VAGUER C. (2008). L'expression de "la totalité" par « complètement» : à propos de « complètement malade ». Des topoï à la théorie des stéréotypes en passant par la polyphonie et l'argumentation dans la langue, Hommages à J.-C. Anscombre (sous la direction de D. Leeman), 363-375.

LENEPVEU V. (2009). «Intégralement » dans le champ des adverbes de complétude ( totalement », « entièrement », « complètement »...). Revue romane, 44, 2, 195-217.

LENEPVEU V. (2013). De la complétude à l'intensité : «totalement», « entièrement» et « complètement ». Langue française, 177, 95-109.

MILNER J.-C. (1978). De la syntaxe à l'interprétation, quantités, insultes, exclamations. Paris : Seuil. RIEGEL M., PELLAT J.-Ch. \& RIOUL R. (1994). Grammaire méthodique du français. Paris : PUF.

${ }^{1}$ Hachette encyclopédique (1994), Larousse-Maxipoche (2012), Le petit Larousse illustré (2012), Le petit Robert (2012), etc.

${ }^{2}$ Ci-dessous quelques exemples pour illustrer le problème chez les sinophones en classe du FLE niveau intermédiaire :

* Léo a les yeux complètement petits.

?? Vous êtes malade. Vous avez tout à fait besoin de repos.

?? Vous êtes tout à fait en retard.

${ }^{3}$ "Le degré complet s'exprime par les adverbes totalement, entièrement, complètement, absolument, tout à fait. » (Le bon usage, §955)

${ }^{4}$ "Correspondant au "superlatif absolu" traditionnel, l'intensité élevée se marque d'abord par l'adverbe très [...], fort [...] et bien [...], ainsi que tout à fait. $\dot{A}$ ces adverbes courts s'ajoute une liste d'adverbes en -ment, [...] absolument, complètement, extrêmement [...]. » (Grammaire méthodique du français, pp.362-363)

${ }^{5}$ Notons que F.-F. Chen ne traite pas du tout complètement, ni tout à fait en termes de degré.

${ }^{6}$ LEEMAN D. \& VAGUER C., op. cit., p.365.

${ }^{7}$ Les adjectifs nettoyable et recyclable sont également non-gradables. Ils sont acceptés par tout à fait, mais aussi par complètement. Nous reviendrons sur ce type d'exemples.

${ }^{8}$ AnsCOMBRE J.-C. (2013: 29).

${ }^{9}$ En ce qui concerne les propriétés qui distinguent qualifiants intrinsèques de qualifiants extrinsèques, cf. ANSCOMBRE J.-C. (1991 ou 2013).

${ }^{10}$ L'un des lecteurs anonymes nous a fait remarquer que la suite C'est complètement minable semble possible. En effet, il s'agit d'un usage très familier, voire sous standard, de complètement qui s'emploie souvent avec des adjectifs à valeur négative, dont nous parlerons plus loin. 
${ }^{11}$ Dans une phrase comme Il a complètement rempli la bouteille, on parle du remplissage de la bouteille : «le remplissage continue jusqu'à ce qu'il n'y ait plus de vide » (CHEN F.F., op. cit., p.9)

12 "Dans son emploi quantitatif (ou extensionnel), l'adverbe [complètement] porte sur un procès télique impliquant un référent susceptible de constituer un tout. » (LENEPVEU V. (2009: 196))

${ }^{13}$ F.-F. Chen a proposé également une caractérisation qualitative pour tout à fait : « il s'agit de la mise en relation de P actualisé avec une image de P provenant de l'énonciateur » (op. cit., p.18). Mais l'approche adoptée par elle s'est basée essentiellement sur l'analyse du contexte, sans recourir à des propriétés linguistiques pour justifier son hypothèse.

${ }^{14}$ LEEMAN D. \& VAGUeR C., op. cit., p.365 et p.369.

15 J.-C. Anscombre n'analyse pas dans son article (2010) les structures autres que être + Adjectif. Nous pensons qu'il est légitime de ranger la phrase La voiture est en panne dans les propriétés extrinsèques, et accidentelles plus précisément.

${ }^{16}$ AnsCOMBRE J.-C. (2010: 195).

17 Selon l'un des lecteurs anonymes, l'enchaînement Ça y est, le café/la soupe est complètement chaud(e), vous pouvez commencer est possible. C'est un bon exemple de la variabilité individuelle de la langue qui complique le travail des linguistes. Signalons au passage un exemple qui semblerait poser moins de problèmes d'acceptabilité chez certains francophones natifs: La soupe est complètement réchauffée.

${ }^{18}$ Exemple emprunté à LEEMAN D. \& VAGUER C., op. cit. Lorsque malade s'accorde avec complètement, l'adjectif ne peut qu'avoir le sens mental.

${ }^{19}$ La soupe n'est pas encore complètement chaude est un énoncé irréprochable. En effet, c'est le marqueur aspectuel pas encore - le contraire de déjà qui indique l'accomplissement — qui rend possible l'enchaînement.

${ }^{20}$ Phénomène observé par D. Leeman \& C. Vaguer.

${ }^{21}$ Selon certains de mes informateurs, La tumeur est tout à fait disparue ou La tumeur a tout à fait disparu sont acceptables même en dehors d'un contexte de confirmation. Ceci n'est pas le cas de *Le chalet est tout à fait réduit en cendres, ni *Le chalet s'est tout à fait consumé. Il est possible que l'on interprète inconsciemment est/a disparu comme « inexistant » en négligeant le processus qui aboutit à l'inexistence.

${ }^{22}$ Cf. KUPFERMAN L. (1992), ANSCOMBRE J.-C. (2000), entre autres.

23 Ernout A. \& Thomas F. (1972: 273-275), cf. aussi Arrivé M., GAdet F. \& GALMICHE M. (1986 : 472).

${ }^{24}$ Nos exemples montrent que les adjectifs participe en -ant susceptibles de se combiner avec complètement sont majoritairement des adjectifs psychologiques, qui désigne en gros le sentiment, l'attitude, l'émotion, etc. On sait depuis un certain temps que les noms/adjectifs psychologiques possèdent des propriétés particulières au sein de la classe générale de noms/d'adjectifs (cf. J.-C. Anscombre (1995), L. Kupferman (1991), D. Leeman (1987, 1995), entre autres). Le fait que les adjectifs psychologiques en -ant aient la possibilité d'être modifiés par complètement pourrait être vu comme une propriété supplémentaire de ce type d'adjectifs.

${ }^{25}$ Les exemples suivants ont été apportés par l'un des lecteurs anonymes que nous remercions :

Je suis, je te l'assure, tout à fait épuisé.

La maison est, je te l'assure, tout à fait nettoyée. 